\title{
\begin{tabular}{l|l} 
POLITIQUES \& & Politiques et management public
\end{tabular}

\section{La certification des comptes des hôpitaux publics à l'horizon 2014 : un état de l'art au travers du témoignage du CHRU de Montpellier}

Implementation of certification of accounts requirements in public hospitals in 2014: an overview based on the experience of the University Hospital of Montpellier

Rodolphe Bourret, Eric Martinez, Maxime Vert, Alexandre Vernhet and Gérald

Naro

\section{OpenEdition}

Electronic version

URL: http://journals.openedition.org/pmp/6909

ISSN: $2119-4831$

Publisher

Institut de Management Public (IDPM)

Printed version

Date of publication: 15 December 2013

Number of pages: 571-581

ISBN: 978-2-7430-1559-6

ISSN: 0758-1726

\section{Electronic reference}

Rodolphe Bourret, Eric Martinez, Maxime Vert, Alexandre Vernhet et Gérald

Naro, « La certification des comptes des hôpitaux publics à l'horizon 2014 : un état de l'art au travers du témoignage du CHRU de Montpellier », Politiques et management public [En ligne], Vol 30/4 | 2013, mis en ligne le 20 avril 2016, consulté le 30 avril 2019. URL : http://journals.openedition.org/pmp/6909 


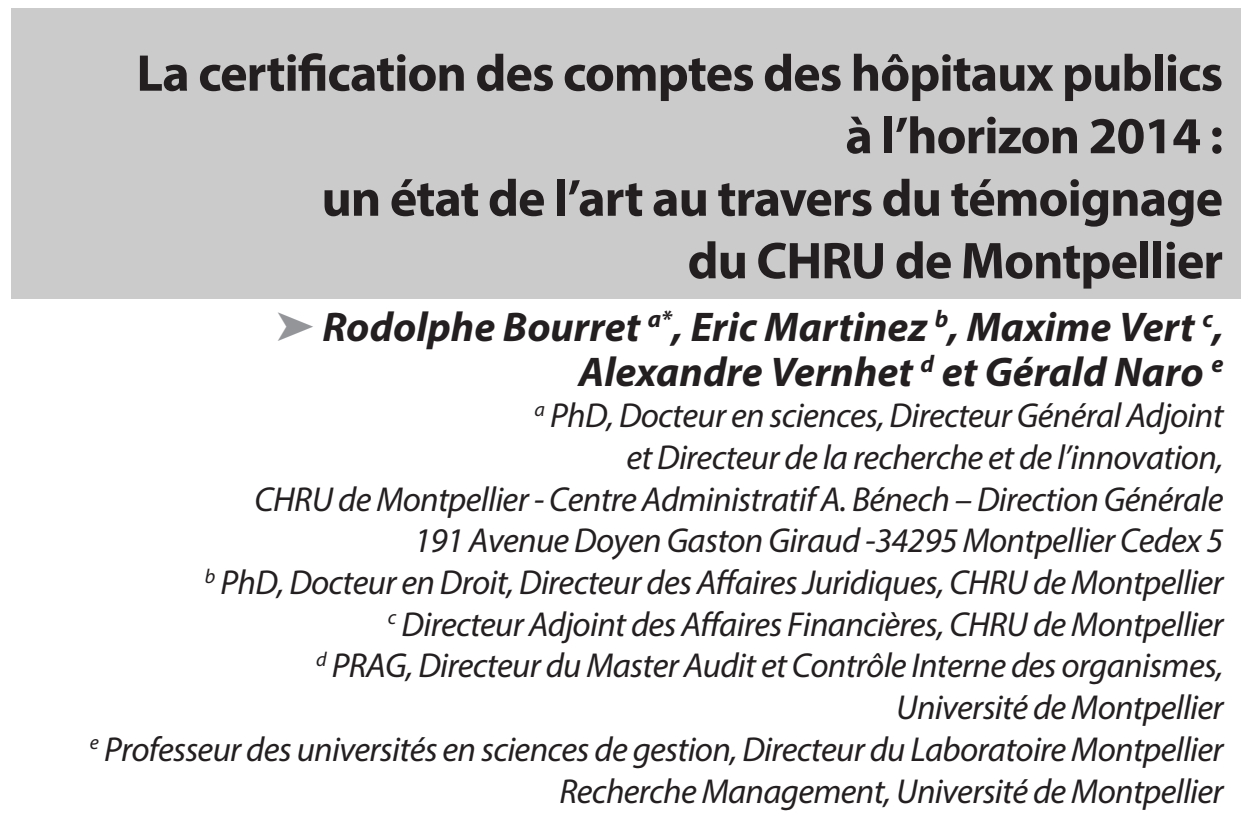

\section{Résumé}

Cette recherche s'intéresse aux nouveaux enjeux portés par la loi « HPST » qui promulgue une obligation nouvelle pour les établissements publics de santé de faire « certifier leurs comptes dans un délai de 4 ans » soit pour la première fois en 2014. Cette disposition, de portée purement technique est au centre de l'un des objectifs majeurs de cette réforme, celui d'optimiser la gestion des hôpitaux publics et de réduire à moyen terme leurs déficits, à tout le moins pour la partie « évitable ». L'étude reprend l'historique depuis 2005 des différentes évolutions comptables. II présente les principaux défis sur le volet «culturel », le volet «procédural » et le volet «comptable». Cette étude est illustrée en fil conducteur dans un des plus grands Centre Hospitalier Universitaire français. Elle montre que malgré les principales zones de risques comptables et managériales connues, l'introduction de cette mesure devrait procurer aux établissements un certain nombre de bénéfices importants.

C 2013 IDMP/Lavoisier SAS. Tous droits réservés

Mots clés : gouvernance, hôpital, certification, management, valeurs publiques.

*Auteur correspondant : r-bourret@chu-montpellier.fr doi :10.3166/pmp.30.571-581 @ 2013 IDMP/Lavoisier SAS. Tous droits réservés 


\section{Abstract}

Implementation of certification of accounts requirements in public hospitals in 2014: an overview based on the experience of the University Hospital of Montpellier. This study focuses on the new challenges created by the HPST (Hospitals, Patients, Health and Territories) law that enacts a new obligation for public health institutions to "certify their accounts within four years", i.e. 2014. This purely technical provision is central to one of the major objectives of the law on hospital reform: optimization of public hospital management and mediumterm deficit reduction. The study reviews the changes in accounting practices since 2005 . It presents the main implementation challenges, perceived from a cultural, procedural, and accounting perspective. Carried out in one of the largest University Hospital Centers in France, this study serves as a guiding thread. It shows that despite known accounting and managerial risk areas, the introduction of this measure should provide institutions with a number of important benefits.

(c) 2013 IDMP/Lavoisier SAS. Tous droits réservés

Keywords: governance, hospital, certification, management, public values.

\section{Introduction}

L'obligation de certification des hôpitaux est une obligation planifiée depuis 2009. En juillet 2009, le président de la République promulgue la loi «Hôpital, Patients, Santé, Territoires $»^{1}$, porteuse de profondes réformes pour le système de soins et l'hôpital public. Cachée au $17^{\mathrm{e}}$ de ses 135 articles, une obligation nouvelle pour les établissements publics de santé apparaît, celle de faire « certifier leurs comptes » dans un délai de 4 ans, soit pour la première fois en 2014. Cette disposition, de portée purement technique, est pourtant au centre de l'un des objectifs majeurs de cette réforme, celui d'optimiser la gestion des hôpitaux publics et de réduire à moyen terme leurs déficits, à tout le moins pour la partie «évitable d de ces derniers. Voyons pourquoi.

Depuis longtemps, les rapports des organismes de contrôle (IGAS, Cour des Comptes) stigmatisent les lacunes de la gestion des hôpitaux publics, relevant pêle-mêle des pratiques contestables en matière de marchés publics ou de gestion patrimoniale, des pertes ou manques à gagner liés à l'inefficience des organisations et des dispositifs de contrôle et, plus généralement, la difficulté pour les établissements à respecter leurs engagements budgétaires. C'est en partant de ce type de constats que les hôpitaux ont été soumis, au cours des années 2000, à la mise en place progressive d'un nouveau processus de gestion et de reporting, notamment financier, vers leurs autorités de tutelle, avec l'instauration de plusieurs dispositifs structurants. Les voici résumés par ordre chronologique :

- 2005 : mise en place de l'EPRD (État des Prévisions de Recettes et de Dépenses) : cadre budgétaire négocié annuellement entre l'établissement et sa tutelle, sur lequel un suivi des réalisations quadrimestriel est fourni l'année suivante par l'Établissement dans le cadre de son obligation de suivi d'activité;

\footnotetext{
' Loi «HPST » dite encore « loi Bachelot » - Loi n 2009-879 du 21/07/2009 portant réforme de l'Hôpital, relative aux patients, à la santé et aux territoires.
} 
- 2006 : publication de l'instruction budgétaire et comptable M21 : référentiel comptable spécifiquement dédié aux Établissements publics de santé. Largement calquée sur le Plan Comptable Général, la M21 consacre l'application de la comptabilité d'engagement et intègre, en outre, un ensemble de dispositions particulières et un plan de comptes spécifiques à l'activité d'un Établissement de soins ;

- 2008 : la Tarification à l'Activité («T2A ») finance à $100 \%$ un premier périmètre d'activité de soins et doit, à terme, s'élargir à d'autres disciplines. Basée sur le suivi quantitatif des actes et séjours médicaux et une nomenclature tarifaire, la T2A se substitue à l'ancien système des dotations globales annuelles dans le financement des activités de soins de Médecine-Chirurgie-Obstétrique (à l'exclusion, temporaire, de la psychiatrie et des Soins de Suite et de Réadaptation).

Ces nouveaux cadres s'ajoutent au mode d'organisation dual des hôpitaux, reposant comme tous les Établissements publics sur une gestion assurée par l'Ordonnateur (Direction de l'hôpital), sous le contrôle d'un Comptable Public garant de la régularité des opérations et de la préservation des actifs et des deniers publics.

Malgré cet attirail réglementaire renforcé et clairement orienté vers une professionnalisation de la gestion des Établissements, les pouvoirs publics, sous la plume de l'IGAS, constataient encore en $2007^{2}$ que « la mise en place de l'EPRD n'avait pas mis fin aux procédures comptables irrégulières déjà relevées par la Cour des Comptes en 2004 et 2005 ». Ces irrégularités étaient nombreuses et potentiellement significatives, puisqu'avaient été, par exemple, relevées dans les comptes des hôpitaux : des créances irrécouvrables non provisionnées, des provisions dimensionnées non pas en fonction des risques encourus mais des capacités budgétaires de l'hôpital à les doter, des charges relatives à des entités juridiquement indépendantes de l'hôpital mais imputées sur son budget, etc.

L'État prenait ainsi conscience du caractère tout à fait vain des efforts de réforme budgétaire en l'absence d'outils de mesure fiables de la performance financière, c'est-à-dire des réalisations effectives censées se comparer aux budgets. Il décidait donc d'engager, à l'instar de ce qui venait d'être mis en place pour les comptes de l'État puis ceux des Universités, une certification des comptes des hôpitaux par un tiers indépendant.

\section{Les enjeux de la certification des comptes des hôpitaux publics}

L'opinion d'audit d'un tiers indépendant vise à donner à l'utilisateur de l'information financière une assurance quant à sa régularité (conformité à la loi et aux règlements), à sa sincérité, à son image fidèle et à sa prudence. Les premiers intéressés par cette appréciation qualitative sont les bailleurs de fonds de l'hôpital que sont les financeurs publics, les financeurs privés de projets de recherche, les collectivités octroyant des subventions, autant d'institutions qui posent - ou poseront - désormais comme condition à leur intervention la présentation d'une comptabilité fiable.

L'autre population demandeuse de comptes certifiés est celle des Établissements de crédit. Dans un contexte de crise financière et de resserrement de l'octroi de crédit de la part des banques, l'accès au financement ne va plus de soi, depuis plusieurs années, pour

\footnotetext{
${ }^{2}$ Rapport de la Commission de concertation « Larcher » sur les missions de l'hôpital, 11/04/2008, page 37.
} 
les Établissements publics, notamment de santé. Comme les autres débiteurs, ils doivent se soumettre à un examen approfondi de leur solvabilité, lequel s'apprécie avant tout sur la base de leurs comptes. Dans ces conditions, il est indispensable pour eux de faire la preuve de la fiabilité de cette matière première analytique, au risque de mettre en jeu leur crédit, et donc rien de moins que leur accès au financement dans les années à venir.

\subsection{Fiabilisation des comptes}

Mais la certification n'est pas une fin en soi pour les pouvoirs publics : ce qui est avant tout recherché à travers cette contrainte, c'est le processus préalable de fiabilisation des comptes, sans lequel bien entendu toute certification s'avérerait impossible. L'objectif sous-jacent est double : fiabiliser les comptes permet, certes, d'obtenir (enfin) une vision réelle du résultat, du patrimoine et de la situation financière des hôpitaux, mais aussi une amélioration des outils de gestion et de pilotage des Établissements, avec à la clé une amélioration attendue de leur efficience.

C'est tout le sens de la circulaire interministérielle du 10 octobre $2011^{3}$, portant diffusion aux Établissements concernés d'un «guide de fiabilisation des comptes des hôpitaux », document dont l'existence même manifestait le constat de l'absence de fiabilité de ces comptes en l'état.

En application de cette circulaire, les Établissements sont invités, dans le délai les séparant de l'échéance de la certification, à :

Procéder à un « balayage exhaustif du bilan », et ceci autant de la part de l'ordonnateur que du comptable public, afin de repérer et corriger les postes en anomalie ou insuffisamment justifiés. L'objectif est de remédier aux erreurs accumulées par le passé, et de présenter, à l'ouverture du premier exercice contrôlé, un bilan exempt de risques comptables majeurs susceptibles d'empêcher la certification ${ }^{4}$;

Optimiser les procédures, les contrôles et les systèmes d'informations pour garantir un niveau de contrôle interne minimal nécessaire à l'auditeur dans le cadre de son approche d'audit par les risques.

\subsection{Acteurs et délais}

La loi HPST a laissé le soin à un décret (non encore paru) de définir qui seront les auditeurs des différents Établissements : Cour des Comptes ou commissaires aux comptes. Il est acquis par ailleurs que l'ensemble des certificateurs appliqueront la démarche normalisée d'audit financier (reposant sur des normes de travail telles que les International Standards on Auditing «ISA» ou les Normes d'Exercice Professionnel « NEP» des commissaires aux comptes).

Les interlocuteurs sollicités par l'auditeur au cours de sa démarche de certification seront multiples et appartiendront aussi bien aux services de l'ordonnateur (Direction Générale, direction financière, pôles médicaux, directions des achats, des ressources humaines et des affaires médicales, des affaires juridiques, de l'informatique...) qu'à ceux du comptable public

\footnotetext{
${ }^{3}$ Circulaire interministérielle nDGOS/DGFIP/PF/PF1/CL1B/2011/391 du 10 octobre 2011.

${ }^{4}$ Il est précisé qu'à titre dérogatoire, les Établissements seront autorisés à enregistrer ces corrections d'erreurs en imputant leur impact sur les capitaux propres, et non sur le résultat de l'exercice de correction.
} 
(opérations de trésorerie, contrôle de conformité, suivi du recouvrement....). C'est en effet l'une des particularités des Établissements publics en matière de production et de contrôle des comptes : les responsabilités sont de facto partagées entre l'ordonnateur et le comptable.

Le destinataire du rapport du certificateur sera le Conseil de Surveillance de l'Établissement; le rapport sera transmis, de plein droit, aux autorités de Tutelle (Agence Régionale de Santé, Cour des Comptes).

En termes de délais, le premier exercice soumis à certification devrait être, au sens de la loi HPST, l'exercice 2014. Compte tenu de la présentation d'une période comparative, le bilan d'ouverture soumis à certification sera donc le bilan du premier jour de l'exercice 2013.

Suivant ce calendrier, nous vivons en ce moment même un exercice dont les comptes seront les premiers soumis à certification. Le compte à rebours est donc lancé pour :

- procéder, au plus tard à l'ouverture de l'exercice 2014, aux corrections nécessaires du bilan d'ouverture;

- mettre en place avant la première intervention de l'auditeur (2014/2015) le cadre de contrôle interne et comptable minimal qu'il est en droit d'attendre.

À ce jour, les hôpitaux ont donc en majorité lancé leur préparation à la certification, en structurant ce vaste chantier en mode projet, et en s'adjoignant le plus souvent les services d'un cabinet d'audit pour les accompagner dans cette démarche.

Le CHRU de Montpellier a, pour sa part, initié la phase active du projet en septembre 2012.

\section{Objectifs et déroulement d'une démarche de certification des comptes}

La certification consistera, pour l'auditeur, à attester sous sa responsabilité du caractère régulier et sincère des comptes, définis par la M21 comme un ensemble formé du bilan, du compte de résultat et de l'annexe.

Pour chacun des comptes significatifs, l'auditeur cherchera à vérifier que les objectifs d'audit sont remplis. On peut les résumer ainsi :

- Existence : les comptes ne présentent que des transactions, des actifs ou des passifs réels (contre-exemple : présentation d'un immeuble à l'actif alors qu'il a été cédé);

- Exhaustivité : les comptes présentent toutes les transactions de la période, tous les actifs et tous les passifs à la clôture (contre-exemple : achats de décembre $\mathrm{N}$ enregistrés en janvier $\mathrm{N}+1)$;

- Exactitude : les montants figurant dans les comptes ne sont pas erronés (contre-exemple : créances en monnaies étrangères non converties au bilan);

- Évaluation : les actifs, passifs et transactions sont correctement évalués au regard de la réglementation relative à l'inventaire (contre-exemple : appareil médical obsolète et sans valeur marchande présenté pour une valeur non dépréciée à l'actif) ;

- Présentation : les transactions, actifs et passifs sont présentés dans les comptes de manière conforme à la présentation requise par la réglementation et en respectant le principe de sincérité (contre-exemple : créances en cours d'encaissement présentées au bilan en trésorerie disponible).

De manière transversale, l'auditeur vérifiera également que les règles de base de la comptabilité sont respectées (principe de prudence, séparation des exercices, coûts historiques, image fidèle, continuité d'exploitation, intangibilité du bilan d'ouverture, même s'il pourra 
y être fait exception pour les corrections d'erreurs à titre exceptionnel avant la certification ${ }^{5}$. La démarche de l'auditeur vise à vérifier le respect de ces principes. Au terme des ses travaux d'audit, il doit s'assurer que les risques d'erreurs ou l'impact chiffré de la contravention à ces règles (montant cumulé des erreurs) restent inférieurs à son seuil de signification ${ }^{6}$.

Suivant la nature de ses conclusions, l'auditeur peut émettre :

- une certification pure et simple;

- une certification avec observation(s), ces dernières pouvant consister à attirer l'attention sur une note importante de l'annexe, sur un changement de méthode comptable ou sur une incertitude;

- une certification avec réserve(s), indiquant son désaccord sur un sujet particulier (par exemple dans le cas de détection d'erreurs significatives non corrigées dans les comptes), ou bien une limitation à ses travaux (par exemple en cas d'impossibilité de se prononcer sur la correcte évaluation d'un actif significatif ou en cas d'insuffisance du contrôle interne...);

- un refus de certifier, pouvant se baser sur une absence d'auditabilité des comptes, des désaccords majeurs sur les principes comptables, ou encore l'inexistence d'un niveau minimal de contrôle interne.

\subsection{Approche par les risques}

La démarche d'audit appliquée par l'auditeur est une approche dite par les risques ${ }^{7}$. Celui-ci va en effet focaliser ses contrôles sur les zones de risques qu'il aura mises en évidence, qui peuvent résulter :

- de risques d'erreurs inhérents à la nature des transactions ou activités, en raison par exemple de leur complexité (cas de la facturation hospitalière) ou de leur nature même (cas des provisions pour litiges, basées sur des estimations);

- de faiblesses dans le dispositif de contrôle interne mis en place par l'Établissement pour gérer les risques de survenance d'erreurs dans ses comptes.

L'auditeur va modéliser l'activité de l'Établissement en processus comptables et financiers $^{8}$, et apprécier pour chaque processus quels sont les risques inhérents et quelle est la qualité du contrôle interne.

La combinaison de l'appréciation « risque inhérent/risque de contrôle interne » met en évidence un certain nombre de zones de risques d'erreurs dans les états financiers (lorsque les risques inhérents et/ou les risques de contrôle interne sont élevés), sur lesquelles il va concentrer son travail de contrôle des comptes par différentes techniques de vérification (retour aux pièces justificatives, confirmation directe de tiers, recalculs, rapprochements, etc.). A contrario, il procédera à des vérifications allégées sur les comptes ou ensembles

\footnotetext{
5 II semblerait même, d'après les discussions en cours avec la DGFIP, que ce régime des corrections d'erreurs puisse être prolongé au-delà de l'entrée en certification. Ce point devra faire l'objet d'un suivi, après avis de l'ensemble des instances de place.

${ }^{6}$ Ce seuil peut se définir comme le montant d'une erreur qui, si elle avait été corrigée, aurait conduit l'utilisateur des comptes, se basant sur ceux-ci, à prendre une décision différente.

${ }^{7}$ Le « risque » se définissant pour l'auditeur comme le risque de présence d'une erreur dans les comptes.

${ }^{8}$ Les outils méthodologiques mis à disposition des hôpitaux par la DGOS et la DGFIP ont modélisé l'activité hospitalière en 6 cycles : achats, immobilisations, paie/personnel, recettes, trésorerie, fonds déposés.
} 
de comptes pour lesquels il aura conclu à l'absence de risque d'erreur importante, en raison de l'absence de risques inhérents significatifs et/ou en présence d'un contrôle interne suffisamment robuste pour prévenir, détecter et corriger les erreurs.

On voit bien que la démarche de certification ne consiste pas à une simple vérification de la justification des soldes comptables, qui ne serait pas suffisante pour atteindre l'ensemble des objectifs d'audit exposés plus haut, notamment l'exhaustivité des transactions et l'ensemble des problématiques d'évaluation.

Au contraire, le cœur de la démarche consiste à décrire, tester et évaluer les procédures mises en place par l'entité pour autoriser, exécuter, contrôler et enregistrer l'ensemble de ses opérations. C'est pourquoi l'existence et la qualité de ce dispositif, communément désigné par le vocable de « contrôle interne », sont des conditions indispensables à l'objectif d'une certification des comptes.

Pour terminer cette description rapide de la démarche d'audit, précisons que l'auditeur demandera, au terme de ses travaux, la signature par les responsables de l'établissement des comptes (probablement au cas d'espèce une signature conjointe de l'Ordonnateur et du Comptable) d'une lettre d'affirmation. Cette lettre a pour but de lui confirmer formellement que les comptes ont été établis de bonne foi, et que l'ensemble des informations nécessaires à leur examen lui ont bien été fournies.

\subsection{Les principales zones de risques comptables connues}

À ce stade de nos travaux de préparation à la certification, en coordination avec le cabinet de conseil que nous avons missionné et avec les autres Établissements concernés ainsi que les différentes parties prenantes (Direction générale des Finances Publiques, Ministère de la Santé, Compagnie Nationale des Commissaires aux comptes), les sujets susceptibles de faire obstacle à la certification dans les hôpitaux sont nombreux et représentent autant de zones d'investigation dans les deux ans à venir.

Citons par exemple et non exhaustivement :

- La justification des actifs immobilisés : réalité, appartenance et correcte évaluation du patrimoine (notamment immobilier) ne sont généralement pas ou peu documentés ;

- L'exhaustivité des passifs : le sous-provisionnement significatif des engagements envers le personnel (notamment les droits à congés accumulés dans les comptes épargne-temps) serait quasi généralisé;

- Le correct rattachement des charges : le « déport» d'enregistrements d'achats sur la période suivante aux fins de respecter les engagements budgétaires est une pratique prohibée, mais connue;

- L'exhaustivité et la correcte évaluation de la facturation hospitalière sont un sujet d'attention majeur car la complexité de la réglementation et des circuits administratifs peut conduire l'Établissement à ne pas appréhender, involontairement, ses droits à recettes et/ou à générer des titres de recettes de mauvaise qualité, qui peuvent par conséquent occasionner des rejets de la part du payeur;

- La correcte affectation des dépenses entre charges et immobilisations : il s'agit d'une thématique sensible (cas rapportés d'activation indue de dépenses incorporelles, notamment en matière de prestations immatérielles en particulier informatiques ou corporelles, comme certains travaux de réparation); 
- La valorisation des créances et le dimensionnement des provisions pour créances douteuses : l'étape du provisionnement n'intervient souvent qu'au seuil de l'irrécouvrabilité, alors qu'elle devrait se concrétiser beaucoup plus tôt, dès qu'un doute sérieux apparaît sur la liquidité d'une créance;

- Le traitement comptable des coûts et des recettes liés aux activités de recherche est souvent incorrect (défaut de symétrie);

- Le provisionnement des risques et charges liés aux litiges ou aux obligations implicites semble très partiel (désamiantage...);

- L'homogénéité des méthodes comptables dans la durée n'apparaît pas toujours garantie (exemple : durées d'amortissement) et certaines méthodes comptables prévues par la M21 ne seraient pas appliquées (exemple : comptabilisation et amortissement des actifs par composants);

- Le traitement comptable de certains instruments financiers complexes (emprunts structurés dits «toxiques ») serait incomplet et négligerait la constatation des risques financiers encourus.

\section{Les principaux défis en matière de contrôle interne}

Au-delà de ces problématiques comptables, les Établissements doivent mettre en conformité leur contrôle interne. Les défis à relever sont, là encore, nombreux. Les hôpitaux souffrent tout d'abord d'un défaut général de formalisation des procédures de contrôle interne comptable et financier. Aussi faudra-t-il dans les mois qui viennent formaliser un corps procédural minimum, qui sera complété, enrichi et mis à jour avec le temps.

À tout le moins, l'Établissement devra pouvoir produire à l'auditeur une documentation lui permettant :

- Au niveau global, de comprendre le cadre général de contrôle interne (organigramme, répartition des pouvoirs de décision, niveaux de délégation et de contrôle, fonctionnement général du système d'informations...);

- Au niveau de chaque processus, de comprendre comment les transactions sont initiées, autorisées, exécutées, contrôlées et enregistrées.

II s'agit d'un travail colossal et compliqué, d'autant qu'il requerra obligatoirement l'implication de nombreux acteurs au sein des différents services fonctionnels (y-compris non financiers), seuls à même de décrire les procédures qu'ils appliquent au sein des différents processus. Une fois les processus existants décrits, il conviendra de les analyser afin d'évaluer leur conception (les procédures décrites sont-elles bien pensées pour répondre aux risques?) et leur fonctionnement (les procédures réellement appliquées sont-elles bien celles qui sont décrites?). Cette analyse pourra mettre en évidence des faiblesses de conception et/ou des cas de mauvais fonctionnement de procédures. Avant l'arrivée de l'auditeur, les mesures correctrices devront être prises pour remédier à ces dysfonctionnements, en commençant par les plus significatifs en termes de risques.

Une autre difficulté en matière de contrôle interne réside dans les systèmes d'informations : peu homogènes entre établissements, souvent très complexes (nombre d'applications et d'interfaces, contraintes de confidentialité des données médicales, échanges de flux 
d'informations avec des éditeurs/hébergeurs extérieurs ou les organismes payeurs...) et insuffisamment sécurisés (problèmes de fiabilité des interfaces, existence de développements spécifiques internes peu documentés, gestion des habilitations laxiste), ils constituent à la fois le cœur du système de production de l'information comptable et l'une de ses principales faiblesses potentielles. La fiabilisation et l'auditabilité des systèmes d'informations sont donc, en soi, un enjeu central de l'objectif de certification.

Le dernier écueil significatif constaté dans beaucoup d'établissements est d'ordre culturel : sitôt la frontière de la direction financière franchie, peu d'acteurs du dispositif de contrôle interne ont conscience de leur rôle dans ce dispositif. Or celui-ci peut être essentiel, même s'il est indirect.

C'est le cas, pour ne citer qu'un exemple, des services médicaux et médico-administratifs qui sont tout à la fois à l'origine des flux de facturation, et responsables de la qualité des données transmises aux organismes payeurs. Peu d'entre eux, pourtant, ont conscience de générer, chaque minute, des écritures comptables. Cette sous-responsabilisation de nombreux «acteurs cachés » de la fiabilité des comptes se trouve renforcée par le manque de culture de l'écrit et de la formalisation des contrôles, ainsi que par le sous-développement d'une réelle fonction de révision comptable au sein des Établissements.

\section{Contraintes du projet et bénéfices collatéraux attendus pour l’hôpital}

La préparation d'un hôpital public à la certification des comptes est compliquée par le fait qu'il évolue dans un univers de contraintes juxtaposées :

- complexité de l'activité ;

- taille et déconcentration des décisions et des actes de gestion au sein de l'établissement ;

- sophistication des circuits informatiques;

- coproduction des comptes par l'ordonnateur et le comptable;

- cadre budgétaire contraignant;

- référentiel comptable (M21) encore mouvant;

- concepts de contrôle interne et de comptabilité peu connus des acteurs;

- prééminence de la logique budgétaire sur la logique comptable;

- risque de résistance au changement de la part de directions déjà sollicitées, par ailleurs, sur de nombreux projets réglementaires ou d'établissement;

- manque d'intérêt pour le sujet de la certification, perçu comme uniquement comptable.

- Pour autant, les efforts déployés dans la conduite de ce projet devraient procurer aux Établissements un certain nombre de bénéfices, au rang desquels :

- l'impact de l'amélioration du contrôle interne sur la performance : sécurisation des flux, indicateurs de gestion plus pertinents et plus exacts, facturation plus exhaustive, détection des anomalies;

- l'obtention d'une meilleure maîtrise du patrimoine et d'une protection des actifs renforcée ;

- une responsabilisation des acteurs accrue;

- les effets positifs, en termes de dynamique humaine, d'un projet d'Établissement, complexe mais fédérateur. 


\section{La démarche de préparation du CHRU de Montpellier}

La préparation de la première certification par le CHRU de Montpellier comporte trois époques, étalées schématiquement sur deux ans :

- le diagnostic de la qualité comptable et du contrôle interne existant (6 mois);

- le traitement des faiblesses mises en évidence dans le diagnostic, sous la forme d'un plan d'actions défini de concert avec tous les acteurs internes concernés (phase actuellement en cours : 1 an);

- et enfin des phases de test et d'exécution, comprenant la finalisation du bilan d'ouverture et l'audit à blanc des derniers comptes annuels ou intermédiaires produits avant l'arrivée du certificateur (6 mois).

Avec 10 établissements, 9 écoles, 12000 agents, 2700 lits et places, et 770 millions d'euros de budget, le Centre Hospitalier Régional Universitaire de Montpellier est le premier employeur de sa région et le $7^{\mathrm{e}}$ Centre Hospitalier Régional Universitaire de France.

Le projet est organisé autour d'un comité de pilotage présidé par le chef d'Établissement et le Directeur Général adjoint en charge des finances. Il repose, dès les premières phases, sur une implication participative de toutes les instances et directions de l'ordonnateur, mais aussi sur une association du comptable public à la démarche.

L'ensemble du projet est conduit avec le concours d'un cabinet d'audit de premier plan rompu d'une part aux techniques de commissariat aux comptes, d'autre part à l'accompagnement de projets.

Au stade actuel de nos travaux, le diagnostic et la définition du plan d'actions sont finalisés et l'Établissement s'apprête à lancer l'exécution de ce plan. En synthèse, nous pouvons retenir que ce projet nécessite de mettre en œuvre des actions coordonnées sur trois axes majeurs :

- un volet «culturel », dont l'objectif est de diffuser des concepts nouveaux dans le domaine du contrôle interne, de la performance financière et des principes comptables ;

- un volet « procédural », qui consiste à formaliser les modes opératoires actuels concourant directement ou indirectement à la préparation des comptes, et à profiter de ce travail descriptif pour corriger ou compléter les procédures et les rendre plus efficientes;

- enfin, un volet « comptable», visant à mettre en conformité les comptes avec la réglementation et les bons usages, une fiabilisation au bénéfice de la pertinence des décisions et de la comparabilité des Établissements entre eux.

Compte tenu de l'étroitesse du délai restant pour conduire le projet, l'Établissement adopte une démarche pragmatique, intégrant une hiérarchisation des enjeux, afin de traiter en priorité les points susceptibles de menacer le plus sérieusement l'objectif de certification.

L'ampleur des actions à entreprendre dans le cadre d'un tel projet dépasse largement un horizon de deux ans, car il suppose, au-delà de travaux techniques étendus, une réelle évolution des mentalités. Sa finalisation fera donc l'objet d'un plan d'actions pluriannuel à compter de l'entrée en certification. 


\section{Bibliographie}

Guide de fiabilisation des comptes des hôpitaux, Ministère du Travail, de l'Emploi et de la Santé (Direction générale de l'offre de soins) - Ministère du Budget, des Comptes Publics et de la Réforme de l'État (Direction Générale des Finances Publiques) - octobre 2011 (www.sante.gouv.fr)

Fiches comptables et cartographie des risques, mêmes auteurs (www.sante.gouv.fr)

Normes d'exercice professionnel des Commissaires aux Comptes - tableau synthétique (https://www.cncc.fr/ sections/documentation_profes/documentation_de_ref/norme_et_doctrine_pr/table_synthetiques_d/table_ des_normes)

Instruction comptable et budgétaire M21 en vigueur - Instruction provisoire consolidée - novembre 2013 (http:// www.collectivites-locales.gouv.fr/m21-instruction-vigueur) 